\title{
Cryogenic fluid level sensors multiplexed by frequency-shifted interferometry
}

\author{
Fei Ye, ${ }^{1, *}$ Tong Chen, ${ }^{2}$ Di Xu, ${ }^{2}$ Kevin P. Chen, ${ }^{2}$ Bing Qi, ${ }^{1}$ and Li Qian ${ }^{1}$ \\ ${ }^{1}$ Department of Electrical and Computer Engineering, University of Toronto, \\ 10 King's College Road, Toronto, Ontario M5S 3G4, Canada \\ ${ }^{2}$ Department of Electrical and Computer Engineering, University of Pittsburgh, \\ 3700 O'Hara Street, Pittsburgh, Pennsylvania 15261, USA \\ ${ }^{*}$ Corresponding author: fei.ye @utoronto.ca \\ Received 15 March 2010; revised 30 June 2010; accepted 23 July 2010; \\ posted 26 July 2010 (Doc. ID 125245); published 3 September 2010
}

\begin{abstract}
We present a liquid level sensing system for cryogenic fluids based on an array of aluminum-coated fiber Bragg gratings written in high-attenuation fibers (HAFs) interrogated by frequency-shifted interferometry (FSI). The sensors are heated up optically through the absorption of light at the core of the HAF sections. The distinct thermal response of sensors in the liquid from that in the gas provides an unambiguous means to detect the liquid level. FSI allows the sensors to have overlapped spectral response, and, therefore, has the potential of accommodating a larger number of sensors in the array. The measurement of liquid nitrogen level using this system was experimentally demonstrated. The successful combination of aluminum-coated HAF Bragg grating sensors and the FSI technique promises a viable solution for liquid level sensor networks at cryogenic temperatures. (c) 2010 Optical Society of America OCIS codes: $\quad 060.2370,120.3180,060.3735,060.4230$.
\end{abstract}

\section{Introduction}

Liquid level sensing has important applications in fields such as chemical processing, fuel storage, and transportation. A variety of techniques have been developed for liquid level sensing, involving methods that are mechanical [1], electrical [2], acoustic [3], or ultrasonic [4], and optical methods [5-16]. Among them, electrical liquid level sensors are widely used in practice. However, if the liquid is corrosive or explosive, the reliability and safety of the sensor will become a major concern. Moreover, for multiple sensors, an electrical sensing system requires intensive wiring, cabling, and feed-throughs, unavoidably increasing the system complexity, cost, and the potential of failure. Fiber-optic liquid level sensors are more advantageous in several regards. Made of dielectric materials, fiber-optic sensors are immune to electromagnetic interference. They are

0003-6935/10/264898-08\$15.00/0

(C) 2010 Optical Society of America also spark free and chemically inert. Another important feature of fiber-optic sensors is their multiplexing capability, which enables one to use a shared light source and detection unit for multipoint measurement, thanks to various fiber-optic components developed for communications.

Many fiber-optic liquid level sensing techniques have been reported. For example, such a sensing system can be based on differential optical absorption [5]. The liquid level can also be determined by directing a collimated light beam onto the liquid surface, and monitoring the reflected light intensity that is dependent on the liquid surface distance [6]. The pressure of the liquid has also been utilized to gauge the liquid level $[7,8]$. But the majority of the fiberoptic techniques rely on the refractive index difference between the liquid and the gas [9-16]. They include techniques based on the principle of total internal reflection [9-12]. The refractive index difference between liquid and gas leads to different critical angles at the interfaces between the sensing element and the surrounding liquid or gas, and the optical 
loss experienced by the light upon reflection at an interface indicates whether the sensing element is in liquid or air. There are also techniques based on the interaction between guided light in a fiber and the surrounding fluid [12-16]. The difference between indices of refraction between liquid and gas gives rise to different mode confinement characteristics in the fiber. Fiber parameters can be chosen such that light suffers high loss at the fiber section in the liquid. The transmitted power of the fiber can, therefore, be used to determine the liquid level. This type of sensing scheme can provide us with information on the length of the fiber section submerged in the liquid, giving one-dimensional liquid level information, but their use is usually limited to liquids whose indices of refraction are within a certain range, and they require large index contrast between gas and liquid. Although the above techniques work well in many circumstances, they may not be suitable for sensing in a cryogenic environment, which is of particular importance in space applications, as well as in the distribution of liquid fuels, such as hydrogen and natural gas. The techniques in $[5,6]$ may not work due to disturbances such as those caused by mist or ripples. Liquid level sensing schemes based on index of refraction may not function effectively at extremely low temperatures, as the index difference between cryogenic fluids and the surrounding gas is rather small [11]. Liquid level sensing is even more demanding in space missions, in which one not only deals with cryogenic temperatures, but an environment that is high-g or micro-g, as well. This prevents the successful application of liquid level sensing schemes based on liquid pressure $[7,8]$. In a micro$\mathrm{g}$ environment, the liquid fuel distribution in a fuel tank is rather complex. One-dimensional level measurement [13-16] in the fuel tank does not provide sufficient information on the fuel distribution. Therefore, there is a need for developing a more comprehensive and robust method of liquid level sensing at cryogenic temperatures.

Ideally, we seek a fiber-optic sensing system that is capable of unambiguously distinguishing liquid and gas at multiple sensing points so as to provide two-dimensional or three-dimensional liquid distribution information in a cryogenic environment. To achieve multipoint sensing, fiber-optic sensing elements in a sensor network need to be interrogated. Conventional fiber-optic sensor multiplexing schemes include time-division multiplexing (TDM) [17], wavelength-division multiplexing (WDM) [18], or a combination of both [19]. In a TDM scheme, pulsed light and a fast detection unit are required. A WDM scheme is limited by the trade-off between sensor number and sensor dynamic range, for each sensor must have a distinct wavelength window. In other words, spectral overlap between sensors is not allowed.

In this paper, we present an all-fiber multipoint liquid level sensing system that can be applied reliably in a cryogenic environment. Based on aluminum- coated high-attenuation fiber Bragg gratings (HAFBGs), the sensors with overlapping wavelength windows in a serial array are interrogated with a continuous-wave (CW) tunable laser and a slow detector, by frequency-shifted interferometry (FSI) $[20,21]$. The liquid sensing principle of this work exploits the different thermal properties of liquid and gas, and is achieved using active fiber Bragg grating (FBG) sensors powered by the in-fiber light [22-24]. These sensors exhibit drastically different sensor responses when heated in liquid and in air. In-fiber heating at extremely low temperatures implies a possibly large sensor Bragg wavelength shift. A temperature swing from near 2 to $300 \mathrm{~K}$ room temperature may induce a sensor Bragg wavelength shift of $\sim 5.7 \mathrm{~nm}$ for a FBG centered at $1550 \mathrm{~nm}$ [25]. FSI is a unique, well-suited fiber-optic sensor multiplexing scheme for this application. Unlike WDM, it allows spectral overlap so that a large number of HAFBG sensors can be interrogated in a serial array within a limited spectral range. FSI needs only a CW tunable laser, a slow detector, and an acousto-optic modulator (AOM). Thus it avoids the pulsed light source and fast electronics required for a TDM scheme. The successful incorporation of the active sensor technology into a FSI-based sensing system provides a novel solution for liquid level sensing applications in a cryogenic environment. We demonstrate the capability of this sensing system by applying it in a multipoint liquid nitrogen level measurement. The same technique can also be applied for liquid level sensing at room temperature [23].

\section{Active HAFBG Sensor Design}

FBG sensors are frequently used as temperature or strain sensors [25]. The change of temperature or applied strain on a FBG can be monitored from the shift of its resonance wavelength (Bragg wavelength). The design of our active HAFBG is shown in Fig. 1. Each sensor consists of a section of $1 \mathrm{~cm}$ bare HAF (INO, 0.2 and $0.5 \mathrm{~dB} / \mathrm{cm}$ at $1550 \mathrm{~nm}$ ), fusion spliced to standard single-mode fibers (Corning SMF-28) at both ends. The spliced fiber then underwent a hydrogen loading process at $2000 \mathrm{psi}$ at room temperature for seven days. A $\mathrm{KrF} 248 \mathrm{~nm}$ excimer laser and a phase mask were used to write a $5 \mathrm{~mm}$ FBG in the HAF section. This was done by exposing the HAFs to $200 \mathrm{~mJ} 3 \mathrm{~ns}$ pulses for 30,000 cycles. To relieve the strain and release the hydrogen, the fiber was baked for $12 \mathrm{~h}$ under $120^{\circ} \mathrm{C}$. UV-curable adhesives were applied to two splices to enhance the ruggedness

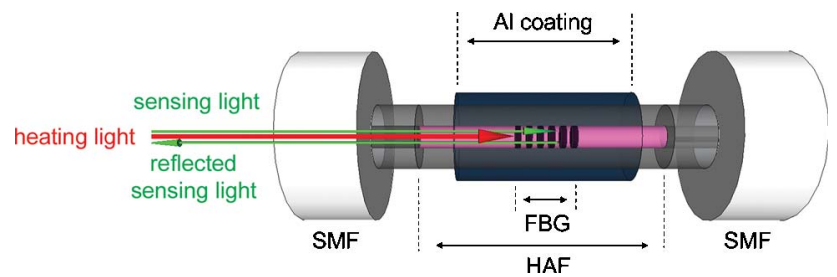

Fig. 1. (Color online) Design of Al-coated HAFBG sensor and its principle of operation. 


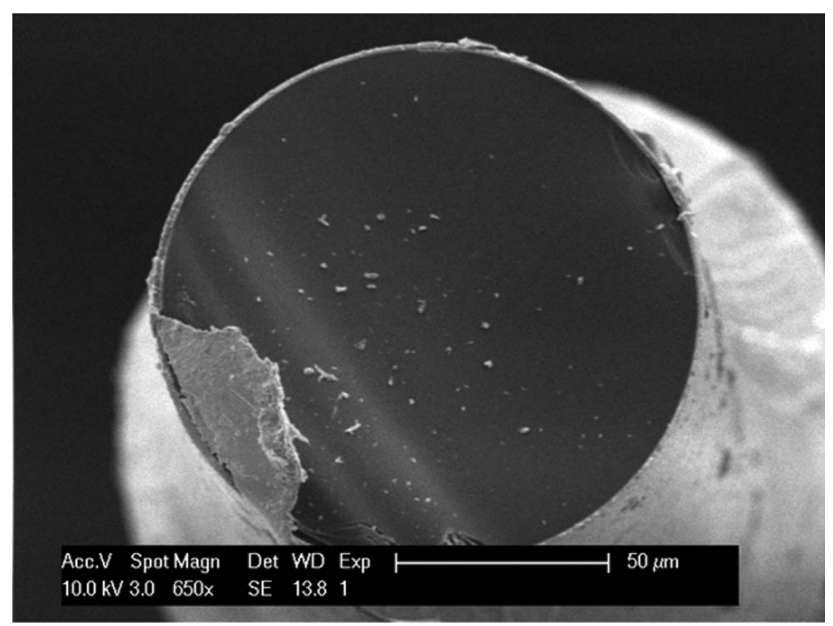

Fig. 2. SEM image of a sample HAFBG sensor's cross section.

of the fiber. The bare HAF section was finally sputter coated with a $150 \mathrm{~nm}$ thick aluminum (Al) film on a rotation stage. A scanning electron microscope (SEM) image of a fiber sensor's cross section is shown in Fig. 2. The cleaved facet shows a layer of $\mathrm{Al}$ coating surrounding the HAF section. The debris, particularly on the bottom left, is due to imperfect cleaving.

The Al-coated FBGs written in HAF can be heated up all-optically by sending light propagating in the fiber core. As the liquid has a much larger specific heat capacity and thermal convection rate than in its gaseous phase, with the same amount of heating power, the temperature change on the sensor submerged in the liquid is much smaller than that in the gas. In terms of the sensor response, the Bragg wavelength shift is small when the sensor is heated in the liquid but much larger when the sensor is heated in the gas. The dramatic spectral response difference exhibited by the heated HAFBGs can be used to identify unambiguously whether the sensor is in liquid or gas.

Compared with previously reported in-fiber heating methods $[23,24]$, our current sensor design has many advantages. In $[23,24]$, the heating of the sensor is produced by the absorption of heating light by the metal coating. The heating light propagates either in the cladding of a SMF [23] or the inner cladding of a double-clad fiber [24]. The heating light is coupled into the sensor array from a high-power diode laser array. The heating efficiency is very low due to low coupling efficiency and loss of heating power in the cladding [23,24]. Furthermore, the heating profile is nonuniform for long sensors, which induces chirp in the grating spectra [22]. The sensor design presented in this paper, on the other hand, offers the flexibility of delivering the heating light power to the sensors through the fiber core. Both the sensing light and the heating light can be fed into the sensor array from a single feed-through. An optical heating source, such as an erbium-doped fiber amplifier (EDFA) can be coupled into the SMF fiber directly. The heating light can travel over long dis- tances with little loss before reaching the sensors, and can interact efficiently with the HAF section. The sensor heating with this design starts from the HAF core, as a result of the absorption of heating light by the cobalt dopant. The heat generated then diffuses and gets absorbed by the Al coating. This produces a more uniform heating profile and solves the issue of sensor spectral chirp found in [23,24]. Another important feature of this design is that the amount of heat released to each sensor can be tailored by adjusting the loss characteristics of the $\mathrm{HAF}$ and the length of the HAF section.

\section{Frequency-Shifted Interferometry}

FSI is a means to obtain simultaneously the location and intensity information of the light signals reflected at various sensor sites [20,21]. As shown in Fig. 3, when a serial array of reflectors is connected to the output coupler $\mathrm{C}_{2}$ of a fiber Mach-Zehnder interferometer (MZI) having a frequency shifter in one arm, each reflector will form a distinct Sagnac-loop path for two counterpropagating beams to interfere at the MZI input coupler $\mathrm{C}_{1}$. The beam going through path $\mathrm{B}$ first and coming back to $\mathrm{C}_{1}$ from path $\mathrm{A}$ travels in a counterclockwise direction, while the beam going through path $\mathrm{A}$ first and coming back to $\mathrm{C}_{1}$ from path $B$ follows a clockwise direction. The frequency shifter, such as an AOM, shifts the frequency of light by an amount of $f$ as the light passes through it. Because, for a reflector $R_{i}(i=1,2,3, \ldots, N)$, the counterclockwise beam acquires the frequency shift earlier than the clockwise beam, there will be a phase difference $\Delta \phi_{i}$ between the two beams at the time when they interfere. This phase difference is given by [21]

$$
\Delta \phi_{i}=2 \pi \frac{n \cdot 2 L_{i}}{c} f
$$

where $n$ is the effective index of refraction of the core, $c$ is the speed of light, and $L_{i}$ is the distance between the reflector and the MZI. The factor of 2 in the numerator accounts for the two passes of light through the fiber between the MZI and $R_{i}$. The differential interference signal $\Delta I$ out of ports 1 and 2 at $\mathrm{C}_{1}$ is a summation of sinusoidal functions of the frequency shift $f$ [21]:

$$
\Delta I \propto \sum_{i=1}^{N} A_{i} \cos \left[2 \pi \frac{2 n L_{i}}{c} f\right]=\sum_{i=1}^{N} A_{i} \cos \left[2 \pi \cdot F_{i} \cdot f\right]
$$

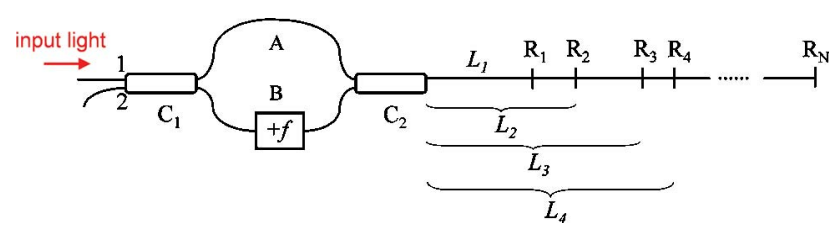

Fig. 3. (Color online) Fiber frequency-shifted Mach-Zehnder interferometer with multiple reflection sites at its output. 
where the relative amplitude is given by

$$
A_{i}(\lambda)=\left[\prod_{j=1}^{i-1} T_{j}^{2}(\lambda)\right] \cdot R_{i}(\lambda) .
$$

Here $T_{j}$ and $R_{i}$ are the transmittance of the $j$ th reflector and the reflectance of the $i$ th reflector, respectively. As the distance between each reflector and the MZI is unique, the sinusoidal functions in Eq. (2) will oscillate at different frequencies $F_{i}=2 n L_{i} / c$ when one sweeps the frequency shift $f$ linearly. A Fourier transform on the interference signal can resolve the signals from the reflectors from $F_{i}$. The Fourier amplitude is proportional to the relative amplitude $A_{i}$, which carries the reflectance information of the $i$ th reflector. By repeating this process for a range of wavelengths, we are able to plot the reflection spectra for each and every reflector. From Eq. (3), we can see that $A_{i}$ conveys the transmittance information of the previous $i-1$ reflectors, as well. A spectral correction can be applied to remove the effect on $A_{i}$ caused by the previous reflectors [21]. In short, the FSI technique can measure the reflection spectra of reflectors (e.g., FBG sensors) in a serial array by addressing them according to their spatial locations. It has been demonstrated that FSI can easily multiplex ten FBG sensors at similar center wavelengths [20]. As the active sensors described in Section 2 are FBGs, they can be conveniently incorporated into a FSI system. A heating source is needed for the active sensor array.

\section{Experiment and Results}

The experimental setup is shown in Fig. 4. It comprises three parts: the heating source, the frequency-shifted interferometer, and the active sensor array. The heating source used in this experiment was an amplified spontaneous emission source that could provide $1 \mathrm{~W}$ in-fiber heating power [26]. The FSI had a fiber MZI formed by connecting two $50 / 50$ wideband fiber couplers, $\mathrm{C}_{1}$ and $\mathrm{C}_{2}$. Typical polarization-dependent loss of the couplers is $\sim 0.1 \mathrm{~dB}$. An AOM (Brimrose) was placed in one arm as the frequency shifter. A tunable laser (Santec TSL-210) was used as the sensing light source. The wavelength resolution of this laser was $10 \mathrm{pm}$, and its output power was set to $4 \mathrm{~mW}$. The differential interference signal was measured by a balanced detector (BD) (New Focus Model 2117) and the output of the BD was fed to a data acquisition board (NI PCI-6251). The data then were processed by a computer. During the experiment, the AOM was swept from 90 to $110 \mathrm{MHz}$ at steps of $0.04 \mathrm{MHz}$ with a step time interval of $1 \mathrm{~ms}$. Each data acquisition cycle was synchronized with an AOM sweep. The fully automated instrument control and data processing were performed by a LabVIEW program on the computer. The heating light from the EDFA and the sensing light from the FSI were coupled into the active sensor array through a 50/50 fiber coupler, $\mathrm{C}_{0}$. The fourth output port of $\mathrm{C}_{0}$ was connected to a power meter (PM) to monitor the heating power. The sensor array was made up of three Al-coated HAFBG sensors of $\sim 4 \%$ reflectivity. The sensors are linked by bare SMF fibers with a standard acrylate jacket.

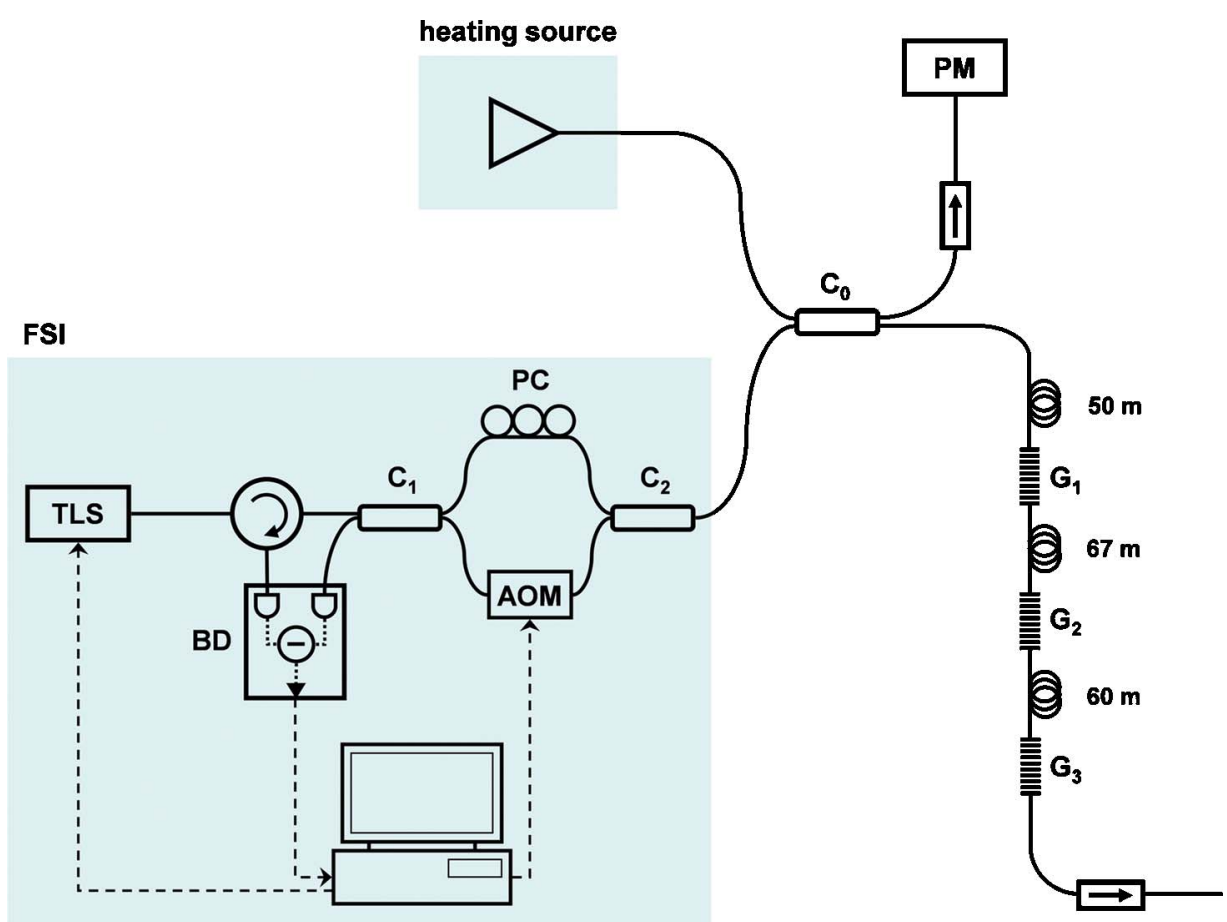

Fig. 4. (Color online) Experimental setup of a liquid level sensing system based on an array of Al-coated HAFBG sensors multiplexed by FSI. TLS, tunable laser source; $\mathrm{BD}$, balanced detector; $\mathrm{C}_{i}, 50 / 50$ fiber directional couplers; PC, polarization controller; AOM, acousto-optic modulator; PM, power meter; $\mathrm{G}_{i}$, Al-coated HAFBG sensors. 
The effect of multiple reflections or the spectral shadowing effect between sensors with such lowreflectivity gratings is small [20]. The counterpropagating beams in the MZI contributed by the reflections from different sensors did not interfere in our experiment, as the fiber lengths between two sensors were each $\sim 10 \mathrm{~m}$ and the tunable laser's coherence control was turned on [21]. The heating light from the EDFA is not coherent and, therefore, it does not interfere with the sensing light. The heating light only introduces a signal at the zero-meter location (a DC signal) in the location-resolved Fourier spectrum, and it does not affect our measurement of sensors. The $1 \mathrm{~cm}$ HAF in the first sensor $G_{1}$ in the array had a loss of $0.2 \mathrm{~dB} / \mathrm{cm}$. Both $\mathrm{G}_{2}$ and $\mathrm{G}_{3}$ had $1 \mathrm{~cm}$ sections of HAF with $0.5 \mathrm{~dB} / \mathrm{cm}$ loss. The total optical power loss for $\mathrm{G}_{1}$ is $\sim 10 \%$, and it is $\sim 15 \%$ for $\mathrm{G}_{2}$ and $\mathrm{G}_{3}$. The higher loss of the last two sensors guaranteed that they would have enough in-fiber heating. To avoid high-power heating light's reflection into the FSI system, an isolator was added at the end of the sensor array, and another was placed between the PM and $\mathrm{C}_{0}$. The fibers between the sensors were wound so that the actual physical separations of the sensors were just 2 or $3 \mathrm{~cm}$.

The sensor array was then lowered into a liquid nitrogen dewar to test their response (Fig. 5). Liquid nitrogen is a frequently used cryogenic fluid whose boiling point is $77 \mathrm{~K}$. The order of the sensors from the top to the bottom was $\mathrm{G}_{1}, \mathrm{G}_{2}$, and $\mathrm{G}_{3}$. The in-fiber heating was kept on during each FSI measurement. The reflection spectra of the three sensors measured by FSI when they were fully immersed in the liquid nitrogen are shown in Fig. 6. As can be seen, without the in-fiber heating (dotted curves in Fig. 6), the center wavelengths of $\mathrm{G}_{1}, \mathrm{G}_{2}$, and $\mathrm{G}_{3}$ are located at $1544.25,1543.06$, and $1543.01 \mathrm{~nm}$, respectively, at $77 \mathrm{~K}$. Note that $\mathrm{G}_{2}$ and $\mathrm{G}_{3}$ 's center wavelengths were

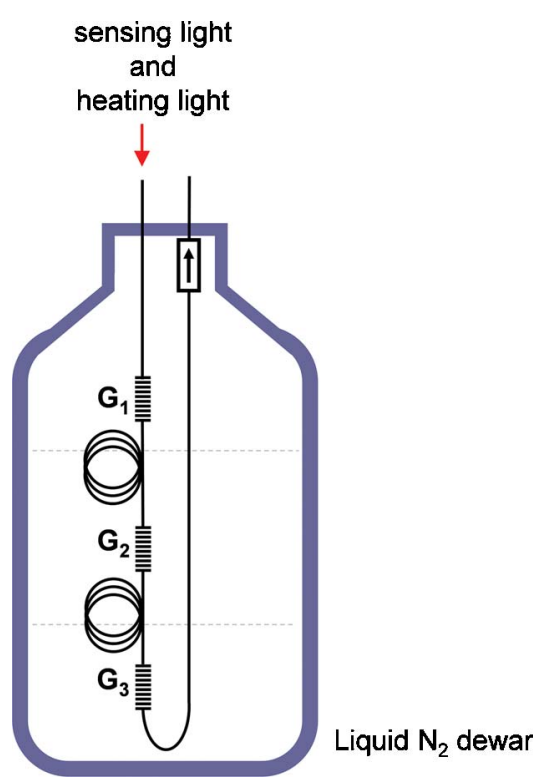

Fig. 5. (Color online) Al-coated HAFBG sensor array in the liquid nitrogen dewar.

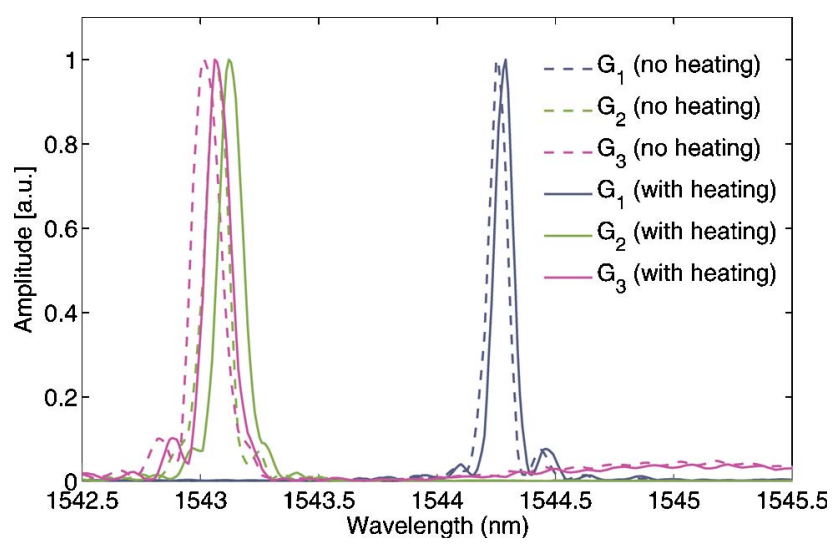

Fig. 6. (Color online) Reflection spectra of Al-coated HAFBG sensors when they were fully emerged in liquid nitrogen measured by FSI (dotted curve, sensor spectra without in-fiber heating; solid curve, sensor spectra with in-fiber heating).

only $0.05 \mathrm{~nm}$ apart, while the full width at halfmaximum (FWHM) of the reflection peaks were $\sim 0.14 \mathrm{~nm}$. Therefore, significant spectral overlap occurred between these two sensors. FSI measurement clearly distinguished the overlapping reflection peaks of $\mathrm{G}_{2}$ and $\mathrm{G}_{3}$, which cannot be easily separated by using an optical spectrum analyzer. When the sensors were heated in the liquid nitrogen (solid curves in Fig. 6), $\mathrm{G}_{1}, \mathrm{G}_{2}$, and $\mathrm{G}_{3}$ exhibited small wavelength shifts of $0.04,0.06$, and $0.05 \mathrm{~nm}$, respectively, which are less than half of the FWHM of the reflection peaks. When the uppermost sensor $G_{1}$ was pulled out of the liquid nitrogen, the FSI measurement showed that the center wavelength of $G_{1}$ was at $1544.79 \mathrm{~nm}$, shifted by $0.50 \mathrm{~nm}$ toward a longer wavelength, compared with the shift in liquid nitrogen [Fig. 7(a)]. This wavelength shift is about 3.5 times larger than the FWHM of the reflection peak. Similarly, when $G_{2}$ was raised out of the liquid nitrogen along with $G_{1}$, its center wavelength was shifted to $1543.91 \mathrm{~nm}, 0.79 \mathrm{~nm}$ larger than when heated in liquid nitrogen [Fig. 7(b)]. Finally, when $\mathrm{G}_{3}$ was pulled above the liquid nitrogen, the center wavelengths of all three sensors experienced large shifts toward longer wavelengths [Fig. 7(c)]. In this case, $\mathrm{G}_{3}$ was centered at $1543.85 \mathrm{~nm}$, shifted by $0.79 \mathrm{~nm}$. Therefore, the heated FBG sensor scheme provides an unambiguous way to determine the liquid level in cryogenic environment. Although the exact experimental conditions, such as sensor temperature, were difficult to repeat in the liquid nitrogen dewar without a temperature feedback control, the sensors' different responses to liquid and air were always reproducible. The sensors in the array always exhibited large Bragg wavelength shifts when heated in the air, and much smaller shifts when heated in the liquid. The sensors did not show deterioration after multiple measurements.

An interesting case occurs when the sensor is placed at the interface of liquid and air, that is, the HAFBG is partially immersed in the liquid. When heated under such condition, the sensor section 

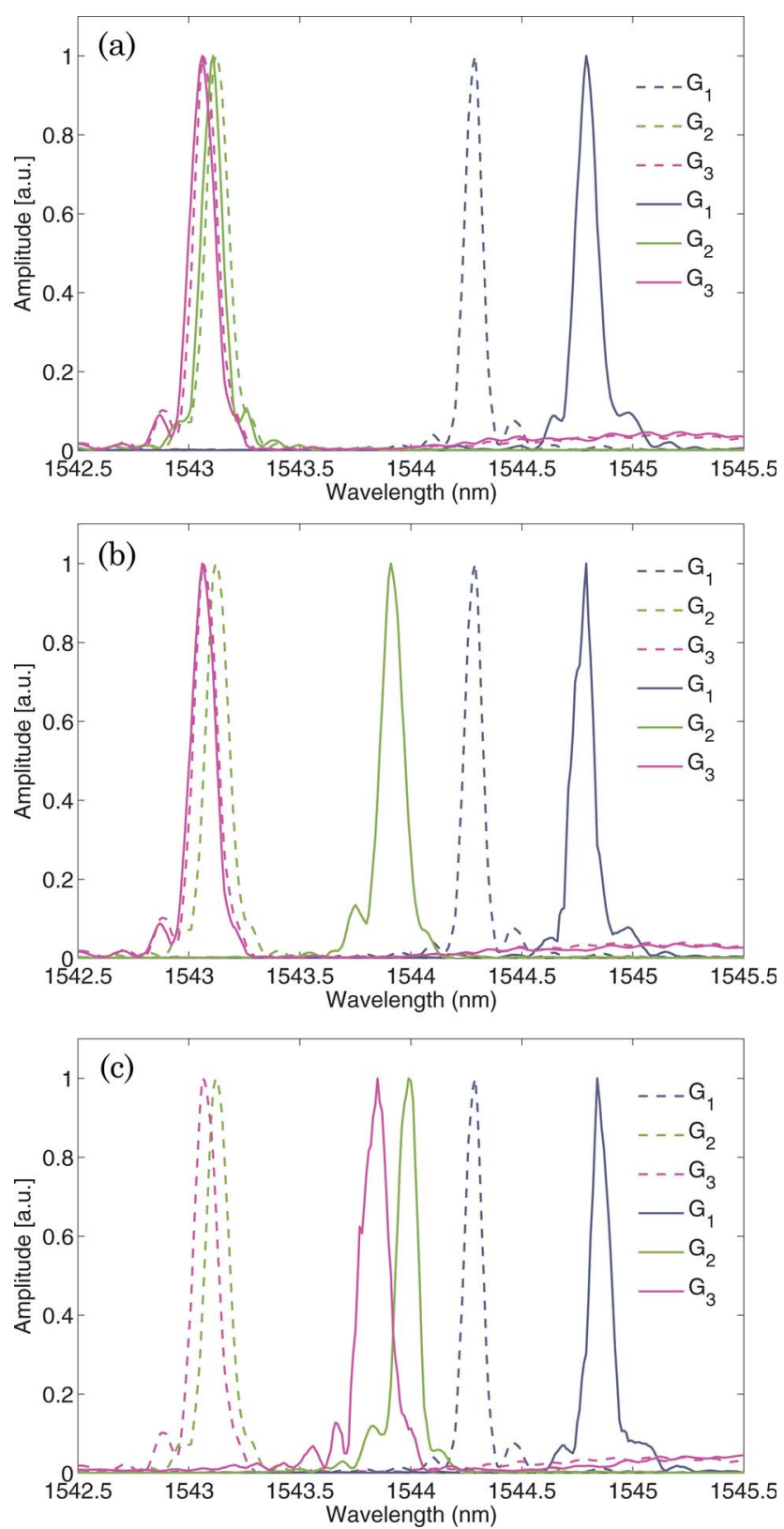

Fig. 7. (Color online) Reflection spectra of heated Al-coated HAFBG sensors as the sensor array was pulled out of liquid nitrogen (dotted curve, sensor reflection spectra measured by FSI when all the sensors were heated in liquid nitrogen). (a) $G_{1}$ was above the liquid nitrogen while $G_{2}$ and $G_{3}$ were in the liquid nitrogen; (b) $G_{1}$ and $G_{2}$ were above the liquid nitrogen while $G_{3}$ was in the liquid nitrogen; (c) all the sensors were above the liquid nitrogen.

exposed to the air will experience much larger temperature rise and expansion than that submerged in the liquid. The sensor essentially becomes two FBGs with different periods. Depending on the length of the sensor in the liquid, the reflection peak of the sensor may broaden, or even split into two. A reflection spectrum of a heated Al-coated HAFBG sensor at the liquid-air interface exhibiting peak split is shown in Fig. 8. The reflection peak at $\sim 1544.58 \mathrm{~nm}$ was induced by the section of HAFBG in the air,

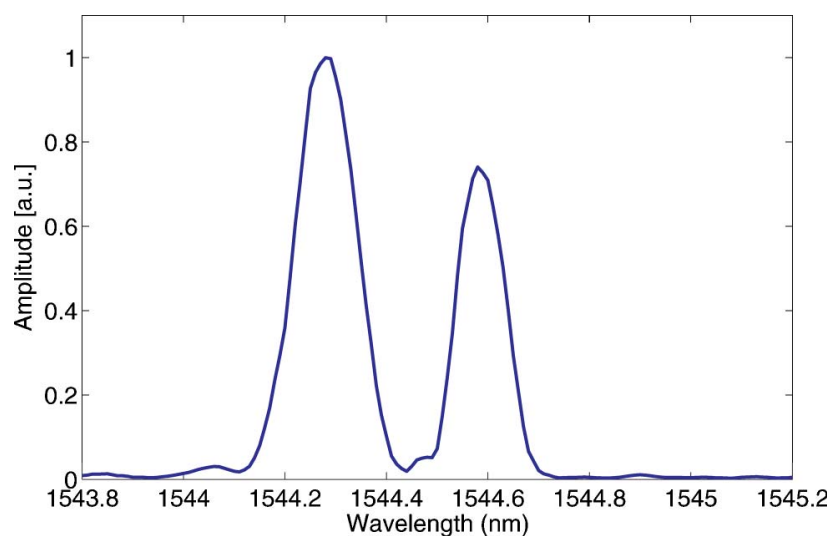

Fig. 8. (Color online) Reflection spectrum of a HAFBG sensor showing a reflection peak split when it is heated at the boundary between liquid nitrogen and air.

while the peak at $\sim 1544.28 \mathrm{~nm}$ was the result of sensor submerged in the liquid. The reflection peak split introduces complications into the spectrum, which are especially undesirable for a sensor array multiplexed by WDM. However, as FSI can resolve the location of the reflection, it is able to reveal that two reflection peaks actually come from the same sensor. Therefore, reflection peak split does not affect the measurements by FSI.

The performance of the heated level sensor array is determined by several factors. First, the Bragg wavelength shift of each sensor is determined by the infiber heating and the thermal response of the sensor. To obtain distinct and uniform spectral responses for different HAFBG sensors in series, both the doping level and the fiber length of each HAF section need to be precisely tailored. For example, in this experiment, the sensors $G_{2}$ and $G_{3}$ down the sensor array are designed to have higher percentages of heat absorption than $G_{1}$, which ensures sufficient thermal response for the later sensors.

The maximum number of sensors employed in a single serial array is limited by the interrogation resolution and the thermal loss on each sensor. The interrogation resolution can be enhanced by using a high-resolution tunable laser and well-written FBGs. In addition, with finer spectral resolution, less power is needed to heat up each sensor for the thermal discrimination between liquid and gas phases, and more sensors can be applied to a single sensor array.

As FBG sensors are temperature dependent, wavelength shift induced by surrounding temperature change may alter the sensor response to local heating. For example, the wavelength shift of $G_{1}$ in Fig. 7(a) was $0.05 \mathrm{~nm}$ shorter than that in Fig. 7(c). This additional wavelength shift was probably caused by a temperature difference of $5 \mathrm{~K}$, since in Fig. $\underline{7(\mathrm{c})} \mathrm{G}_{1}$ was closer to the dewar opening and farther away from the liquid nitrogen. This problem can readily be solved by attaching a conventional non-HAF FBG sensor to the HAFBG and calibrating the temperature dependence. 
Polarization drift may also affect the measurement results. When the two counterpropagating beams reflected from a sensor interfere, the visibility of the fringe depends on the polarization. It is maximized when the two beams have the same polarization. Therefore, a polarization controller (PC) was used to optimize the visibility of the interference fringe. If the polarization fluctuates significantly during the measurement, the interference fringe visibility change may cause noise in the measured reflection spectra. Under our current experimental conditions, adjustment of the PC was not needed during the entire measurement, indicating negligible polarization drift.

The resolution of our liquid level sensing system, or the capability of accurately identifying the liquid level, is determined by the length of the HAFBG sensor (5 mm in our experiment). Physically closely spaced sensors can be used to monitor the liquid level with high resolution. Note that the physical spacing of the sensor, at least in one dimension (such as height), is not limited by the spatial resolution of the FSI technique itself, since the fiber in between two sensors can be wound into a small spool and the two sensors can even overlap in height (if they are placed with a lateral shift).

The measurement time of the system is a function of the experimental parameters used. We tune the laser wavelength in steps of $\lambda_{\text {step }}$ after every full AOM sweep over a frequency range $\Delta f$. The wavelength tuning step time is negligible compared with the AOM sweep time over $\Delta f$. The interference data were processed at each wavelength, with a processing time of $T_{\text {process. }}$. The measurement time $T_{\text {measure }}$ can, therefore, be calculated as

$$
T_{\text {measure }}=\left(\tau \cdot \frac{\Delta f}{f_{\text {step }}}+T_{\text {process }}\right) \cdot \frac{\Delta \lambda}{\lambda_{\text {step }}},
$$

where $\tau$ is the AOM frequency sweep step time interval, and the AOM frequency sweep step and the tunable laser tuning range are denoted by $f_{\text {step }}$ and $\Delta \lambda$, respectively. Currently, $\tau$ is limited to about $1 \mathrm{~ms}$, due to the slow driver electronics of the AOM. $T_{\text {process }}$ is slightly less than $0.5 \mathrm{~s}$ on a slow computer. Depending on the sensor array configuration, $\Delta f$, $f_{\text {step }}, \Delta \lambda$, and $\lambda_{\text {step }}$ can be carefully chosen in order to minimize the measurement time. For example, if we use $\Delta f=15 \mathrm{MHz}, f_{\text {step }}=0.1 \mathrm{MHz}, \Delta \lambda=$ $3 \mathrm{~nm}$, and $\lambda_{\text {step }}=0.03 \mathrm{~nm}$, the total measurement time is about $1 \mathrm{~min}$. However, improvement on the AOM driver hardware can potentially increase the AOM sweep speed by 2-3 orders of magnitude, and the data processing can be accelerated by another 2-3 orders of magnitude by using dedicated hardware. So a measurement time of $\sim 100 \mathrm{~ms}$ is achievable. Furthermore, a suitable choice of $\Delta f$ and $f_{\text {step }}$, depending on the required spatial resolution and sensing range [21], can be used to optimize the measurement time.

\section{Conclusion}

In summary, we measured the liquid level for a cryogenic fluid, using an array of Al-coated HAFBG sensors along a single fiber multiplexed by FSI. Each sensor in the array is heated uniformly through the absorption of optical power in the core of the HAF section. The distinct spectral responses of the heated sensor in the liquid and in the air serve as a clear indicator of the liquid level. With the FSI technique, multiple Al-coated HAFBG sensors with overlapping Bragg wavelengths can be interrogated in a single fiber feed-through. By precisely controlling the heating profiles of each sensor, a larger number of HAFBG sensors can be incorporated into the sensing network and be measured simultaneously. The demonstration in this work provides a practical solution for the multipoint detection of cryogenic liquid in extreme temperature and gravity conditions.

This work was supported by the Natural Science and Engineering Research Council of Canada, the Canada Research Chairs Program, the Canadian Institute for Photonic Innovations, and the National Science Foundation (NSF) (NSF0644681).

\section{References}

1. B. W. Northway, N. H. Hancock, and T. Tran-Cong, "Liquid level sensors using thin walled cylinders vibrating in circumferential modes," Meas. Sci. Technol. 6, 85-93 (1995).

2. D. K. Hilton, J. S. Panek, M. R. Smith, and S. W. Van Sciver, "A capacitive liquid helium level sensor instrument," Cryogenics 39, 485-487 (1999).

3. R. Osborne, M. Ward, and K. Dawkins, "A micro-machined acoustic sensor array for fuel level indication," Sens. Actuators A, Phys. 115, 385-391 (2004).

4. V. E. Sakharov, S. A. Kuznetsov, B. D. Zaitsev, I. E. Kuznetsova, and S. G. Joshi, "Liquid level sensor using ultrasonic Lamb waves," Ultrasonics 41, 319-322 (2003).

5. C. P. Yakymyshyn and C. R. Pollock, "Differential absorption fiber-optic liquid level sensor," J. Lightwave Technol. 5, 941946 (1987).

6. C. Vázquez, A. B. Gonzalo, S. Vargas, and J. Montalvo, "Multisensor system using plastic optical fibers for intrinsically safe level measurements," Sens. Actuators A, Phys. 116, 22-32 (2004).

7. T. Guo, Q. Zhao, Q. Dou, H. Zhang, L. Xue, G. Huang, and X. Dong, "Temperature-insensitive fiber Bragg grating liquidlevel sensor based on bending cantilever beam," IEEE Photonics Technol. Lett. 17, 2400-2402 (2005).

8. T. Lü, Z. Li, D. Xia, K. He, and G. Zhang, "Asymmetric FabryPerot cavity fiber-optic pressure sensor for liquid-level measurement," Rev. Sci. Instrum. 80, 033104 (2009).

9. A. Wang, M. F. Gunther, K. A. Murphy, and R. O. Claus, "Fiberoptic liquid-level sensor," Sens. Actuators A, Phys. 35, 161164 (1992).

10. P. Raatikainen, I. Kassamakov, R. Kakanakov, and M. Luukkala, "Fiber-optic liquid-level sensor," Sens. Actuators A, Phys. 58, 93-97 (1997).

11. K. E. Romo-Medrano and S. N. Khotiaintsev, "An optical-fibre refractometric liquid-level sensor for liquid nitrogen," Meas. Sci. Technol. 17, 998-1004 (2006).

12. A. A. Kazemi, C. Yang, and S. Chen, "Fiber optic cryogenic liquid level detection system for space applications," Proc. SPIE 7314, 73140A (2009). 
13. S. Khaliq, S. W. James, and R. P. Tatam, "Fiber-optic liquidlevel sensor using a long-period grating," Opt. Lett. 26, 1224-1226 (2001).

14. B. Yun, N. Chen, and Y. Cui, "Highly sensitive liquid-level sensor based on etched fiber Bragg grating," IEEE Photonics Technol. Lett. 19, 1747-1749 (2007).

15. S. M. Chandani and N. A. F. Jaeger, "Optical fiber-based liquid level sensor," Opt. Eng. 46, 114401 (2007).

16. M. Lomer, J. Arrue, C. Jauregui, P. Aiestaran, J. Zubia, and J. M. López-Higuera, "Lateral polishing of bends in plastic optical fibres applied to a multipoint liquid-level measurement sensor," Sens. Actuators A, Phys. 137, 68-73 (2007).

17. R. S. Weis, A. D. Kersey, and T. A. Berkoff, "A four-element fiber grating sensor array with phase-sensitive detection," IEEE Photonics Technol. Lett. 6, 1469-1472 (1994).

18. S. Abad, M. López-Amo, F. M. Araújo, L. A. Ferreira, and J. L. Santos, "Fiber Bragg grating-based self-referencing technique for wavelength-multiplexed intensity sensors," Opt. Lett. 27, 222-224 (2002).

19. G. A. Cranch and P. J. Nash, "Large-scale multiplexing of interferometric fiber-optic sensors using TDM and DWDM," J. Lightwave Technol. 19, 687-699 (2001).
20. F. Ye, L. Qian, Y. Liu, and B. Qi, "Using frequency-shifted interferometry for multiplexing a fiber Bragg grating array," IEEE Photonics Technol. Lett. 20, 1488-1490 (2008).

21. F. Ye, L. Qian, and B. Qi, "Multipoint chemical gas sensing using frequency-shifted interferometry," J. Lightwave Technol. 27, 2449 (2009).

22. K. P. Chen, L. J. Cashdollar, and W. Xu, "Controlling fiber Bragg grating spectra with in-fiber diode laser light," IEEE Photonics Technol. Lett. 16, 1897-1899 (2004).

23. K. P. Chen, B. McMillen, M. Buric, C. Jewart, and W. Xu, "Selfheated fiber Bragg grating sensors," Appl. Phys. Lett. 86, 143502 (2005).

24. M. Buric, K. P. Chen, M. Bhattarai, P. R. Swinehart, and M. Maklad, "Active fiber Bragg grating hydrogen sensors for alltemperature operation," IEEE Photonics Technol. Lett. 19, 255-257 (2007).

25. A. D. Kersey, M. A. Davis, H. J. Patrick, M. LeBlanc, K. P. Koo, C. G. Askins, M. A. Putnam, and E. J. Friebele, "Fiber grating sensors," J. Lightwave Technol. 15, 1442-1463 (1997).

26. T. Chen, M. P. Buric, K. P. Chen, P. R. Swinehart, and M. Maklad, "Active fiber hydrogen sensors for lowtemperature operation," in Conference on Lasers and Electro-Optics (OSA, 2009), paper CThE4. 